\title{
Synaptic overgrowth, hyperconnectivity may define autism subtype
}

\section{BY ANGIE VOYLES ASKHAM}

18 NOVEMBER 2021

A form of autism marked by a distinctive pattern of brain overconnectivity arises from an excess of synapses, according to a new study in mice. The findings bolster the theory that autism subtypes have different biological roots and may require different treatment approaches.

Autistic people have altered brain connectivity, previous work suggests, although it has been unclear whether the strength of the connections are increased or decreased compared with nonautistic people, and where - or even why - the differences occur.

Different mouse models of autism have distinct patterns of brain connectivity, according to a study published earlier this year, a finding that could explain some of the inconsistencies seen in people. But the research group, led by Alessandro Gozzi, senior researcher at the Istituto Italiano di Tecnologia in Rovereto, Italy, wanted to go a step further.

"We found that there are different signatures," Gozzi says. "And now we're trying to decode them."

For the new study, Gozzi and his colleagues examined the brain connectivity of mice that lack a copy of the gene TSC2. These mice model tuberous sclerosis complex, a condition often accompanied by autism. TSC2 typically acts as a brake for the mTOR pathway, which is involved in cell growth and proliferation. Damaging mutations in the gene lead to overactivity of the pathway and an overabundance of synapses across the brain, previous studies have shown.

The new work suggests that mTOR overactivity, and the resulting surplus of synapses, causes a specific pattern of brain hyperconnectivity seen in some autistic people. The findings were published in October in Nature Communications.

\section{Connecting dots:}




\section{Spectrum | Autism Research News}

https://www.spectrumnews.org

Gozzi and his colleagues used functional magnetic resonance imaging (fMRI) to map synchronous activity across the brain in the TSC2 mice. Regions that activate or inactivate in sync are thought to be connected in a circuit.

Compared with wildtype mice, the TSC2-deficient mice show hyperconnectivity in a circuit involving the prefrontal cortex, insular cortex and striatum - three brain areas implicated in autism.

The TSC2 animals did not have atypical white matter structure or any other visible changes to the brain that could explain this connectivity difference. But they had an increased density of dendritic spines, the nodes where a neuron receives signals from other neurons, Gozzi and his colleagues found. Increased spine density also occurs in the brains of some autistic people.

The animals' hyperconnectivity disappeared after the researchers treated the mice with rapamycin, a drug that inhibits the mTOR pathway. And the treated mice no longer showed repetitive grooming or decreased sociability, behaviors reminiscent of those seen in people with autism.

"It's implicating these extra synapses in this hyperconnectivity signature," Gozzi says.

The same hyperconnectivity signature appears in a subset of autistic people, Gozzi and his colleagues found using anonymized fMRI data from the Autism Brain Imaging Data Exchange (ABIDE). The most highly connected regions in this subset also express a disproportionate number of genes that are associated with autism and are capable of interacting with mTOR or TSC2 proteins.

The team also identified other connectivity signatures among the scans. One group of autistic people showed widespread underconnectivity and another showed weak frontal hyperconnectivity.

\section{Signature style:}

"These are very beginning steps, but very important steps," says David Sulzer, professor of psychiatry, neurology and pharmacology at Columbia University, who was not involved in the study.

"It's an important building block in an overall movement to understand what autism really is [and] why it develops," he says. "It's all pointing to problems in normal maturation of brain development," such as the lack of synaptic pruning that takes place when the mTOR pathway goes unchecked.

The TSC-deficient mice are not an ideal model for autism, as not all people missing one copy of the gene are autistic, says Peter Crino, professor and chair of neurology at the University of Maryland in Baltimore, who was not involved in the work. 


\section{Spectrum | Autism Research News}

https://www.spectrumnews.org

A better approach might be to genetically engineer the mice to carry human variants of the gene known to be associated with autism, he says, in which case, "you're going to get a much more realistic approximation of what's happening in in the human brain."

But for now, the results suggest that inhibiting the mTOR pathway is a potential treatment for, at the very least, a subset of autistic people, Crino and Sulzer both say.

Gozzi and his colleagues plan to investigate whether any of the other signatures of atypical connectivity - which may represent different autism subtypes - can be linked to other changes in biology.

Cite this article: https://doi.org/10.53053/QQHB1099 